\title{
Left Ventricular Volumes, Mass and Function normalized to the body surface area, age and gender from CMR in a large cohort of well- treated Thalassemia Major patients without myocardial iron overload
}

\author{
Antonella Meloni ${ }^{*}$, Maria Chiara Dell'Amico', Brunella Favilli', Giovanni D Aquaro', Pierluigi Festa', \\ Elisabetta Chiodi ${ }^{2}$, Stefania Renne ${ }^{3}$, Maria Concetta Galati ${ }^{4}$, Leonardo Sardella ${ }^{5}$, Petra Keilberg ${ }^{1}$, Vincenzo Positano ${ }^{1}$, \\ Massimo Lombardi ${ }^{1}$, Alessia Pepe
}

From 2011 SCMR/Euro CMR Joint Scientific Sessions

Nice, France. 3-6 February 2011

\begin{abstract}
Introduction
Cardiovascular Magnetic Resonance (CMR) allows an accurate and reproducible quantification of left ventricular (LV) parameters. In Thalassemia major (TM) patients have been reported different "normal" LV values due to chronic anemia and eventually pre-existing iron burdens. Moreover, in this population it is unknown the influence of sex and age on LV parameters and no ranges of normal have been reported using MASS $^{\circ}$ software. The aim of this study was to establish the ranges for normal LV volumes, mass and ejection fraction normalized to the influence of body surface area(BSA), age and sex from CMR in a large cohort of well-treated TM patients without myocardial iron overload.
\end{abstract}

\section{Materials}

Among the 923 TM patients who underwent CMR within the MIOT network for the assessment of cardiac iron overload, function and fibrosis, we selected 142 patients with no known risk factors or history of cardiac disease, normal electrocardiogram, no myocardial fibrosis and no myocardial iron overload (all the cardiac segments with a normal T2* value). All patients had been

\footnotetext{
1"G. Monasterio" Foundation and Institute of Clinical Physiology, CNR, Pisa, Italy Full list of author information is available at the end of the article

regularly transfused and chelated since early childhood. Moreover, we studied 71 healthy subjects matched for age and sex. LV function parameters were quantitatively evaluated in a standard way by SSFP cine images using MASS $^{\circledR}$ software. LV end-diastolic volume, end-systolic volume, stroke volume, and mass were normalized to BSA (EDVI, ESVI, SVI, massI).

\section{Results}

Table 1 shows the comparison of the CMR parameters with differentiation for sex and age in TM patients and healthy subjects and the cut-off of normality defined as mean - 2 standard deviation (SD). TM patients showed significantly lower BSA than the controls $(\mathrm{P}<0.0001)$. Significantly higher EDVI and SVI were found only for males 30 years. Significantly higher LVEF were found only for males < 14 years. In TM patients all LV volumes indexes were significantly larger in males than in females $(\mathrm{P}<0.0001$ in all cases). The EF was not different between the sexes. In males the ESVI and the EF were significant different among the age groups $(\mathrm{P}=0.006$ and $\mathrm{P}=0.001$, respectively). In females no significant differences were detected among the age groups.

\section{Conclusion}

In a large cohort of well-treated TM patients significant differences in LV parameters compared to controls were 
Table 1

\begin{tabular}{|c|c|c|c|c|c|c|c|c|c|c|c|c|c|c|c|}
\hline & & $<14$ & & & $14-20$ & & & 20-30 & & & $30-40$ & & & $>=40$ & \\
\hline & $\mathrm{TM}$ & $\mathrm{H}$ & $P$ & TM & $\mathrm{H}$ & $P$ & $\mathrm{TM}$ & $\mathrm{H}$ & $P$ & TM & $\mathrm{H}$ & $P$ & TM & $\mathrm{H}$ & $P$ \\
\hline Males & $N=7$ & $N=7$ & & $N=6$ & $N=6$ & & $N=25$ & $N 1=15$ & & $N=23$ & $N=11$ & & $N=6$ & $N=5$ & \\
\hline EDVI $\left(\mathrm{ml} / \mathrm{m}^{2}\right)$ & $\begin{array}{l}94 \pm 18 \\
(58)\end{array}$ & $75 \pm 11$ & 0.034 & $\begin{array}{l}96 \pm 20 \\
(56)\end{array}$ & $91 \pm 20$ & 0.715 & $\begin{array}{l}103 \pm 17 \\
(69)\end{array}$ & $\begin{array}{l}101 \pm \\
13\end{array}$ & 0.686 & $\begin{array}{l}92 \pm 15 \\
(62)\end{array}$ & $80 \pm 11$ & 0.222 & $\begin{array}{l}94 \pm 9 \\
(76)\end{array}$ & $75 \pm 11$ & 0.013 \\
\hline ESVI (ml/m2) & $\begin{array}{l}31 \pm 6 \\
(19)\end{array}$ & $24 \pm 6$ & 0.058 & $\begin{array}{l}36 \pm 8 \\
(22)\end{array}$ & $35 \pm 14$ & 0.760 & $\begin{array}{l}38 \pm 8 \\
(22)\end{array}$ & $39 \pm 9$ & 0.676 & $\begin{array}{l}32 \pm 6 \\
(20)\end{array}$ & $29 \pm 6$ & 0.160 & $\begin{array}{l}29 \pm 6 \\
(17)\end{array}$ & $28 \pm 10$ & 0.816 \\
\hline $\mathrm{SVI}\left(\mathrm{ml} / \mathrm{m}^{2}\right)$ & $\begin{array}{l}63 \pm 14 \\
\text { (35) }\end{array}$ & $51 \pm 7$ & 0.05 & $\begin{array}{l}57 \pm 12 \\
\text { (33) }\end{array}$ & $56 \pm 8$ & 0.868 & $\begin{array}{l}65 \pm 10 \\
(45)\end{array}$ & $62 \pm 9$ & 0.433 & $\begin{array}{l}59 \pm 10 \\
\text { (39) }\end{array}$ & $51 \pm 10$ & 0.027 & $\begin{array}{l}64 \pm 8 \\
(48)\end{array}$ & $47 \pm 8$ & 0.007 \\
\hline Mass I $\left(\mathrm{g} / \mathrm{m}^{2}\right)$ & $\begin{array}{l}57 \pm 7 \\
(43)\end{array}$ & $68 \pm 5$ & 0.007 & $\begin{array}{l}57 \pm 13 \\
\text { (31) }\end{array}$ & $71 \pm 7$ & 0.043 & $\begin{array}{l}66 \pm 12 \\
(42)\end{array}$ & $77 \pm 12$ & 0.006 & $\begin{array}{l}62 \pm 12 \\
\text { (38) }\end{array}$ & $66 \pm 10$ & 0.184 & $\begin{array}{l}69 \pm 16 \\
\text { (36) }\end{array}$ & $74 \pm 11$ & 0.536 \\
\hline EF (\%) & $\begin{array}{l}66 \pm 4 \\
(58)\end{array}$ & $54 \pm 6$ & $<0.0001$ & $\begin{array}{l}60 \pm 2 \\
(56)\end{array}$ & $66 \pm 14$ & 0.322 & $\begin{array}{l}63 \pm 3 \\
(57)\end{array}$ & $62 \pm 6$ & 0.520 & $\begin{array}{l}65 \pm 3 \\
(59)\end{array}$ & $65 \pm 8$ & 0.972 & $\begin{array}{l}68 \pm 6 \\
(56)\end{array}$ & $62 \pm 9$ & 0.234 \\
\hline Females & $N=2$ & $N=2$ & & $N=8$ & $N=6$ & & $N=24$ & $N=6$ & & $N=33$ & $N=8$ & & $N=8$ & $N=5$ & \\
\hline EDVI $\left(\mathrm{ml} / \mathrm{m}^{2}\right)$ & $\begin{array}{l}53 \pm 8 \\
(47)\end{array}$ & $62 \pm 4$ & 0.951 & $\begin{array}{l}81 \pm 8 \\
(65)\end{array}$ & $80 \pm 9$ & 0.866 & $\begin{array}{l}83 \pm 16 \\
(51)\end{array}$ & $78 \pm 9$ & 0.499 & $\begin{array}{l}77 \pm 11 \\
(55)\end{array}$ & $79 \pm 10$ & 0.669 & $\begin{array}{l}82 \pm 19 \\
(44)\end{array}$ & $77 \pm 16$ & 0.614 \\
\hline ESVI (ml/m2) & $\begin{array}{l}23 \pm 1 \\
(21)\end{array}$ & $22 \pm 8$ & 0.823 & $\begin{array}{l}30 \pm 6 \\
(18)\end{array}$ & $31 \pm 3$ & 0.789 & $\begin{array}{l}30 \pm 8 \\
(14)\end{array}$ & $30 \pm 4$ & 0.883 & $\begin{array}{l}26 \pm 6 \\
(14)\end{array}$ & $29 \pm 7$ & 0.216 & $\begin{array}{l}26 \pm 8 \\
(12)\end{array}$ & $28 \pm 12$ & 0.974 \\
\hline $\mathrm{SVI}\left(\mathrm{ml} / \mathrm{m}^{2}\right)$ & $\begin{array}{l}40 \pm 7 \\
(26)\end{array}$ & $41 \pm 4$ & 0.919 & $\begin{array}{l}49 \pm 3 \\
(43)\end{array}$ & $49 \pm 6$ & 0.892 & $\begin{array}{l}53 \pm 9 \\
(35)\end{array}$ & $48 \pm 6$ & 0.234 & $\begin{array}{l}51 \pm 6 \\
(39)\end{array}$ & $50 \pm 8$ & 0.915 & $\begin{array}{l}54 \pm 11 \\
(32)\end{array}$ & $49 \pm 6$ & 0.394 \\
\hline Mass I $\left(\mathrm{g} / \mathrm{m}^{2}\right)$ & $\begin{array}{l}34 \pm 3 \\
(28)\end{array}$ & $59 \pm 19$ & 0.296 & $\begin{array}{l}47 \pm 8 \\
(31)\end{array}$ & $56 \pm 6$ & 0.040 & $\begin{array}{l}53 \pm 9 \\
(35)\end{array}$ & $54 \pm 12$ & 0.893 & $\begin{array}{l}52 \pm 9 \\
(34)\end{array}$ & $55 \pm 13$ & 0.465 & $\begin{array}{l}51 \pm 12 \\
\text { (27) }\end{array}$ & $56 \pm 5$ & 0.344 \\
\hline EF (\%) & $\begin{array}{l}62 \pm 3 \\
(57)\end{array}$ & $46 \pm 17$ & 0.388 & $\begin{array}{l}63 \pm 4 \\
(55)\end{array}$ & $62 \pm 4$ & 0.694 & $\begin{array}{l}65 \pm 5 \\
(55)\end{array}$ & $62 \pm 3$ & 0.137 & $\begin{array}{l}66 \pm 5 \\
(56)\end{array}$ & $63 \pm 7$ & 0.271 & $\begin{array}{l}66 \pm 4 \\
(58)\end{array}$ & $63 \pm 7$ & 0.254 \\
\hline
\end{tabular}

limited to males $<14$ years and $>30$ years. Appropriate "normal" reference ranges normalized to BSA, sex and age should be used to avoid misdiagnosis of cardiomiopathy in TM patients.

\section{Author details}

"G. Monasterio" Foundation and Institute of Clinical Physiology, CNR, Pisa, Italy. ${ }^{2}$ Arcispedale "S. Anna", Ferrara, Italy. ${ }^{3}$ P.O. "Giovanni Paolo II", Lamezia Terme, Italy. ${ }^{4}$ A.O. "Pugliese-Ciaccio", Catanzaro, Italy. ${ }^{5}$ A.S.L. BA - Osp. "Di Venere", Bari, Italy.

Published: 2 February 2011

doi:10.1186/1532-429X-13-S1-P305

Cite this article as: Meloni et al:: Left Ventricular Volumes, Mass and Function normalized to the body surface area, age and gender from CMR in a large cohort of well-treated Thalassemia Major patients without myocardial iron overload. Journal of Cardiovascular Magnetic Resonance 2011 13(Suppl 1):P305.

\section{Submit your next manuscript to BioMed Central} and take full advantage of:

- Convenient online submission

- Thorough peer review

- No space constraints or color figure charges

- Immediate publication on acceptance

- Inclusion in PubMed, CAS, Scopus and Google Scholar

- Research which is freely available for redistribution

Submit your manuscript at www.biomedcentral.com/submit 